UVX 2010 (2011) 203-207

DOI: $10.1051 /$ uvx/2011029

(C) Owned by the authors, published by EDP Sciences, 2011

\title{
The X-ray calibration for the Laser Integration Ligne and the LaserMégaJoule facilities
}

\author{
S. Hubert and V. Prévot \\ Commissariat à l'Énergie Atomique, DAM, CESTA, BP. 2, 33114 Le Barp, France
}

\begin{abstract}
The Laser Integration Line located at CEA-CESTA is equipped with X-ray plasma diagnostics using different kinds of X-ray components such as filters, mirrors, crystals, detectors and cameras. To guarantee LIL measurements, detectors such as X-ray cameras must be regularly calibrated close to the facility. A new X-ray facility is currently available to perform these absolute X-ray calibrations. This paper presents the X-ray tube based High Energy X-ray Source (HEXS) mainly used to calibrate X-ray cameras and also X-ray filters of plasma diagnostics.
\end{abstract}

\section{THE HEX SOURCE AND THE EXPERIMENTAL SET UP}

The X-ray calibration STATIX laboratory located at the CEA-CESTA, near Bordeaux, provides now the HEX source, a multi-anodes X-ray tube based continuous source developed by the Optique and Électronique Appliquée company consisting of an electron gun and an anode barrel being equipped with a different material $(\mathrm{Al}, \mathrm{Ti}, \mathrm{Cr}, \mathrm{Cu}, \mathrm{Mo}, \mathrm{Ag})$ to obtain characteristic lines.

The X-ray detectors calibration with HEXS is essentially performed with characteristic radiation lines provided by current-voltage set and anode material. The emission spectra are monitored by means of a CANBERRA Ultra LeGe germanium liquid-nitrogen cooled spectrometer. The Ge spectrometer acts as a photon counter and the charge collected by the Ge crystal is converted and processed by preamplifier/amplifier, analogic-digital coder and multichannel analyser stages. The X-ray beam is collimated by a $50 \mu \mathrm{m}$ tungsten diameter collimator to prevent spectrometer saturation. The energy calibration of the Ge spectrometer is achieved by means of X-ray intensity calibrated radioactive sources $\left({ }^{55} \mathrm{Fe},{ }^{65} \mathrm{Zn},{ }^{87} \mathrm{Sr}\right.$ and $\left.{ }^{109} \mathrm{Cd}\right)$. The efficiency of the Ge spectrometer is now determined by calculation including manufacturer specifications such as the Ge dimension and the thickness of the aluminium polarizing layer and enables the X-ray intensity measurement of the HEXS sources in terms of photons per second and per steradian. As the HEXS do not have any monochromating system at the time of the present work X-ray filters of same material as the anode are used to enhance the spectral purity of the sources. The emission spectrum consists of characteristic X-ray lines and a continuous Bremsstrahlung radiation. Typical working intensities of the X-ray sources range from $5.10^{10}$ to $4.10^{11} \mathrm{ph} . \mathrm{s}^{-1} \cdot \mathrm{sr}^{-1}$ with spectral purities lying between $2 \%$ and $8 \%$.

The design of the calibration bench integrating the HEXS source uses the rotating feature of the anode barrel. The anode barrel rotation enables the X-ray beam to be sent either towards the Ge spectrometer for X-ray intensity measurements (the X-ray Intensity Measurement Arm or XIMA) or towards the X-ray detector for calibration purposes (the X-ray detector Calibration Arm or XCA), as illustrated in figure 1.

The HEXS calibrates regularly X-ray streak cameras, X-ray framing camera, X-ray CCD, filters and more recently XRD photodetectors. For all these calibrations we mainly perform spectral sensitivity, energy yield, spatial homogeneity and resolution, quantum efficiency and transmission measurements.

This is an Open Access article distributed under the terms of the Creative Commons Attribution-Noncommercial License 3.0, which permits unrestricted use, distribution, and reproduction in any noncommercial medium, provided the original work is properly cited. 


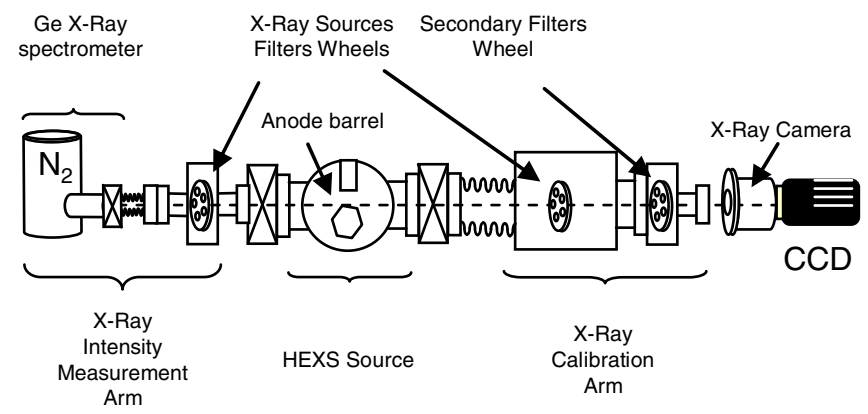

Figure 1. HEXS calibration bench for X-ray detectors and cameras of the LIL.

\section{CALIBRATION OF X-RAY STREAK CAMERA}

The LIL X-ray streak cameras (XRSC) are composed of a P552X Photonis opened tube with a CsI X-ray photocathode working in transmission. The opened tube feature enables the nature of the photocathode to be changed according to the requirement of the LIL experiments. These cameras are typically used over an energy range from $100 \mathrm{eV}$ to $10 \mathrm{keV}$. They can be fitted with 5 different sweep calibres such as $25,10,5,2$ and $1 \mathrm{~ns}$ with a typical minimum temporal resolution of $6 \mathrm{ps}$. The spectral efficiency measurement is carried out in the XRSC static mode. The visible image emitted by the phosphor is recorded by means of a calibrated visible Roper Scientific fibered and backilluminated CCD. The XRSC's energy yield or efficiency is defined as the ratio of visible energy emitted by the phosphor screen to the incident X-ray energy. The XRSC are regularly equipped with photocathode made by Luxel Corporation with lexan, aluminium and CsI layers. Figure 2 illustrates typical spatial homogeneity of new photocathodes with good (a) and bad (b) spatial homogeneity.

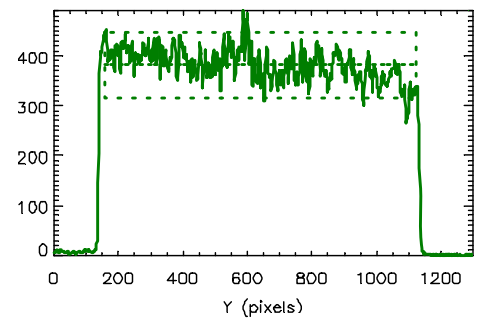

(a)

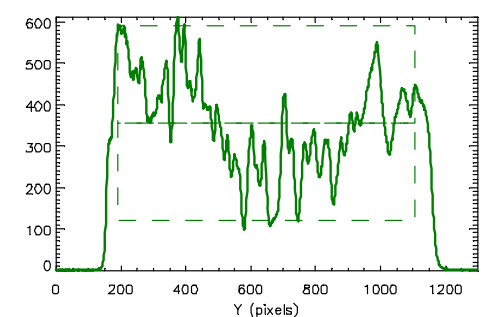

(b)

Figure 2. Examples of different spatial homogeneity of CsI photocathode (cross-section along the observed electronic line).

These examples show that the spatial homogeneity measurement is of great interest before setting the photocathode into the XRSC.

Figure 3 shows recent results of energy yield measurements [1] performed with a hard x-ray photocathode (5000 ̊ lexan layer). We observe that experimental points are in disagreement with the Henke's transmission model [2] at low energy. The solid curve in fair agreement with our experimental points was obtained using the reflection Henke's model with Al as well as the CsI mass absorption cross-section. Previous works showed that the crystallite structure of the CsI layer is not a continuous layer as usually considered in models [3]. This behavior difference between our measurements and Henke's model could be due to the crystallite spherical shape of the CsI. Our experimental results seem 


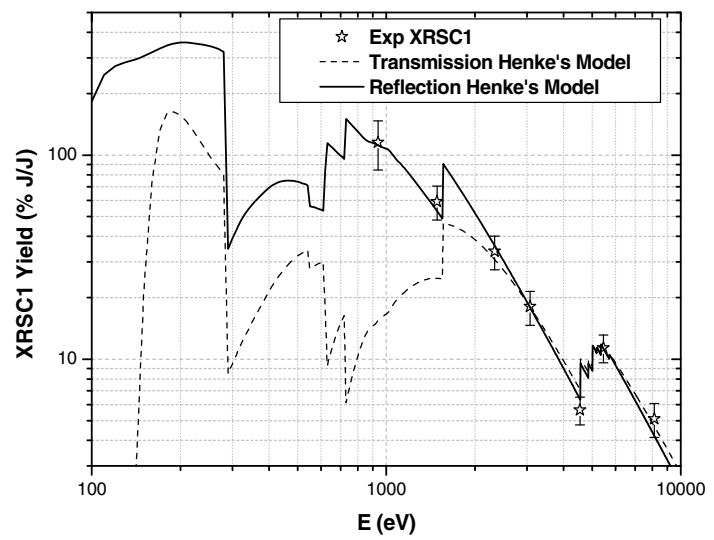

Figure 3. The curves illustrate the Henke model applied for CsI photocathodes in both modes : reflection and transmission. For energies below $3 \mathrm{keV}$, the CsI photocathode appears to work in the "reflection" mode.

to indicate that for soft X-rays incident photons primary electrons are generated on the surface of the spherical crystallite.

\section{CALIBRATION OF X-RAY FRAMING CAMERA (XRFC)}

These cameras consist of a $40 \mathrm{~mm}$ diameter micro channel plate (MCP) device situated $500 \mu \mathrm{m}$ from a phosphor screen. The photocathode is made of line-shaped coating layers on the MCP. For X-ray framing camera calibration the MCP and the phosphor screen are continuously polarized. Figure 4 shows a typical image recorded by the CARIX camera with the four striplines. The CARIX spectral efficiency is determined by means of a visible backilluminated fibered Roper Scientific CCD camera. In the same way as the XRSC case, the CARIX efficiency is defined as the ratio of visible energy emitted by the phosphor screen to incident X-ray energy. Figure 5 shows an example of the efficiency curve recorded for the LIL CARIX camera over the HEXS 929-8900 eV spectral range on which the CARIX theoretical response is superimposed.

To understand and validate our measurements we developed a numerical model which describes the behavior of CARIX using the CARIX MCP geometry [4]. The result curve shows a good agreement between experimental data and theoretical response. The $\mathrm{K}_{\alpha}$ silicon absorption edge at $1839 \mathrm{eV}$ is well reproduced by the model as well as the $\mathrm{M}_{V}$ gold absorption edge at $2206 \mathrm{eV}$. Except for $\mathrm{L}_{\alpha}(937 \mathrm{eV})$ and $\mathrm{K}_{\alpha}(8128 \mathrm{eV}$ : weighted energy) $\mathrm{Cu}$ lines the agreement is relatively good. One can emphasize that the yield is rising around $8 \mathrm{keV}$ which has already been observed on synchrotron [4]. Measurements show that at $8 \mathrm{keV}$ we observe $1 \%$ of direct $\mathrm{x}$-rays which, injected in our model enables to obtain the solid curve which is in better agreement with our data.

\section{CALIBRATION OF X-RAY CCD}

X-ray CCDs are widely used with LIL plasma diagnostics such as X-ray imaging and X-ray spectrometry. At the LIL facility we mainly find X-ray CCD manufactured by Andor Technology (DH436 CCD) and by Roper Scientific (PIXIS CCD and SX-NTE $1300 \times 1340$ EMB CCD). For these kinds of X-ray cameras, absolute quantum efficiency $(\mathrm{QE})$ and spectral sensitivity are of great interest for physicists. To achieve accurate measurements we used an X-ray spectrometer (called SHREX for Spectromètre à Haute RÉsolution X) as a monochromator to increase the spectral purity of the HEXS. This X-ray spectrometer is identical to the SXDHR-1T spectrometer [5] used at the LIL facility and works with either a Johanson concave cylindrical germanium crystal or a beryl one manufactured 


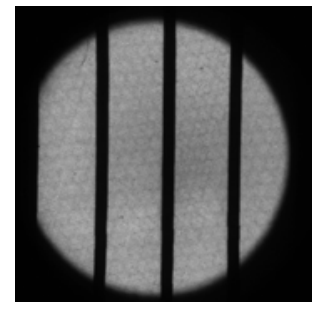

Figure 4. Typical image recorded by the LIL $\mathrm{x}$-ray framing camera (called CARIX).

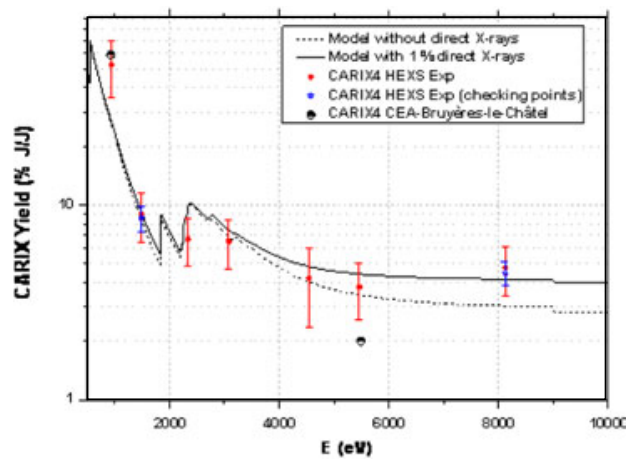

Figure 5. CARIX yield measurements.

by Saint Gobain Crystal Detector. To determine the QE of X-ray CCD this germanium crystal was previously calibrated by Reverdin's team on a $25 \mathrm{kV}$ X-ray generator [5]. X-ray CCD QE measurements performed with SHREX gave rise to very high purity spectra which led to measured QE values expected for this kind of X-ray CCD as illustrated in the following figure 6.

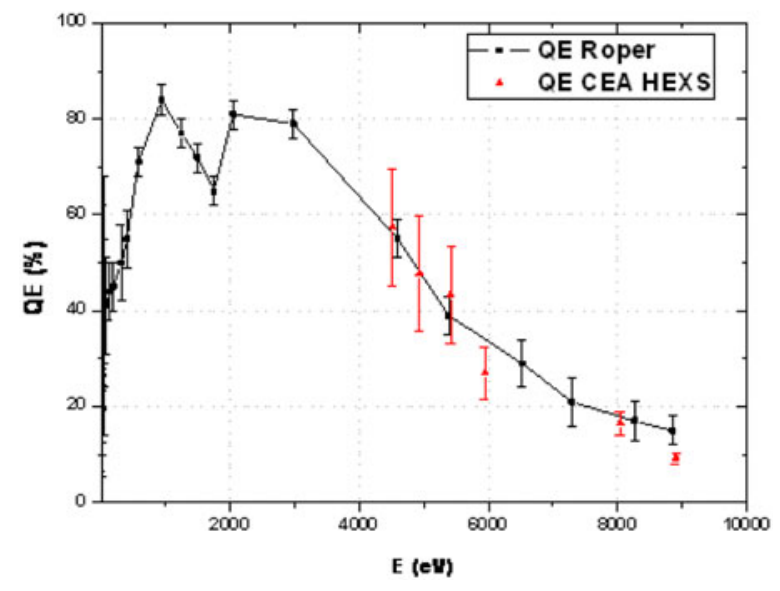

Figure 6. X-ray CCD Quantum efficiency performed on Roper Scientific CCD. The curve is that measured by Roper Scientific with squared plots. Triangular plots are those measured on HEXS bench with the SHREX Spectrometer.

\section{CONCLUSION}

In this paper we have presented the HEXS facility which aims at calibrating regularly all X-ray cameras used on LIL plasma diagnostics and on LMJ soon. By means of a Ge X-ray spectrometer which enables monitoring of HEXS X-ray intensity, absolute X-ray calibrations can be performed. Through several examples we have shown that an X-ray tube based source like HEXS is a powerful tool for X-ray calibration. This facility is now in operation since 2 years with a relatively stable set-up providing increasingly quantitative results on different kinds of cameras. These results are in good agreement with theoretical models and with comparative measurements made on synchrotron by the CEA-DAM 
of Arpajon [6] or by manufacturers. HEXS facility can propose now to users to calibrate several kinds of x-ray detectors. The next monochromator project intending to achieve monochromatic spectra will contribute to perform more accurate measurements on x-ray cameras.

\section{References}

[1] The $\mathrm{x}$-ray calibration facility of the laser integration line in $0.9-10 \mathrm{keV}$ range : the high energy X-ray source and some applications, S. Hubert et al., Rev. Sci. Instr. 81 Issue 8 (may 2010)

[2] Henke B. L., Knauer J. P., Premaratne K, J. Appl. Phys., 52(3), (1981) 1509

[3] On the surface morphology of thin alkali halide photocathode films, T. Boutboul et al., Nucl. Instr. Meth. Phys. Res. A 438 (1999) 409

[4] Features and performances of the "CARIX" MCP framing camera, D. Gontier et al., SPIE 2869 (1997) 82

[5] X-ray calibration of the time resolved crystal spectrometer SXDHR-1t of the Ligne d'Intégration Laser, C. Reverdin et al., Rev. Sc. Instr., 7510 (2004) 3730

[6] X-ray detector calibration at CEA/Bruyères-le-Châtel, L. Beck and al., Nucl. Instr. Meth. Phys. Res. A 369 (1996) 401 